\title{
COMUNICACIÓN
}

\section{DETERMINACIÓN DE OCRATOXINAS EN MAÍZ UTILIZADO EN GRANJAS PORCINAS DE LIMA}

\author{
Omar Salcedo R. ${ }^{1}$,Teresa Arbaiza F. ${ }^{2}$, Orlando Lucas A. ${ }^{2}$ y Antonio Ampuero B. ${ }^{3}$
}

\section{Ochratoxin contamination in yellow corn pig feeds in Lima.}

Peru produces and imports yellow corn (Zea mays) for use as a principal component in pig feed. This cereal is susceptible to ochratoxin toxin produced fungus contamination during storage and handling. Pigs are extremely sensitive to the presence of this toxin which causes nephropathy and immune-suppression, resulting in significant economic losses and posing a danger to public health through consumption of meat from infected animals or the cereal itself. In order to determine the level of ochratoxin contamination in the yellow corn supply, a total of 62 random samples were taken from ships $(n=2)$, markets $(n=20)$ and farms $(n=40)$ and evaluated using ELISA test. Of these samples, $27.42 \%$ exceeded $5 \mathrm{ug} / \mathrm{kg}$ (ppb), the maximum level permitted in some countries, but none of the samples exceeded $50 \mathrm{ug} / \mathrm{kg}$ the maximum set in other countries. These results indicate a risk of Ochratoxin contamination exists in the Peruvian yellow corn supply.

Key words: Ochratoxin, nephropathy, pigs.

Palabras clave: Ocratoxina, nefropatía, cerdos.

En la actualidad el Perú cuenta con una población porcina de 2'787 533, predominando la crianza tecnificada.

La alimentación del cerdo, tiene como alimento básico de su ración el maíz. Este cereal es suceptible para la producción de micotoxinas, entre ellos la ocratoxina A, que atenta contra el óptimo rendimiento y conversión alimenticia del cerdo. Esta toxina ocasiona efectos nefrotóxicos e inmunotóxicos, con las consiguientes pérdidas económicas.

Estudios realizados en países de Europa revelaron que una alta contaminación de los alimentos por ocratoxina $\mathrm{A}$, no sólo ocasiona daño en la industria porcina, sino

\footnotetext{
'Práctica privada.

${ }^{2}$ Laboratorio de Nutrición Animal- FMV-UNMSM

${ }^{3}$ Laboratorio de Producción Animal - FMV-UNMSM
}

que también peligro para la salud pública por su posible asociación con la nefropatía endémica de los Balcanes.

El Perú produce e importa maíz amarillo, por lo que es necesario evaluar el nivel de contaminación por ocratoxina, debido a que en nuestro país no existen estudios ni reglamentos sobre el nivel máximo permitido de ocratoxina en los alimentos.

Por lo tanto, el objetivo del presente trabajo es evaluar el nivel de contaminación por ocratoxina en el maíz amarillo y comprobar si excede las cantidades máximas permitidas en otros países, a fin de emitir recomendaciones. 
El estudio se realizó en el laboratorio de Bioquímica Nutrición y Alimentación_Animal de la Facultad de Medicina Veterinaria Universidad Nacional Mayor de San Marcos, entre los meses de enero y mayo.

Se utilizaron 62 muestras de maíz amarillo (Zea maiz) variedad indurata tomadas al azar, divididas de la siguiente manera:

- 40 muestras de granjas porcinas de Lima.

- 20 muestras de los principales mercados mayoristas de Lima

- 10 muestras del mercado mayorista de la Parada.

- 05 muestras del mercado mayorista de Caquetá.

- 05 muestras del mercado mayorista de Santa Anita.

- 02 muestras de barcos cargueros.

- 01 muestra de un barco proveniente de USA.

- 01 muestra de un barco proveniente de Argentina.

La detección de ocratoxinas, se realizó mediante la prueba de ELISA (Enzyme linked immunosorbent assay), para la lectura de las absorbancias de cada pozo se empleó un lector automático de ELISA con filtro de $650 \mathrm{~nm}$ (Bird y Felman, 1996).

Los resultados obtenidos en las pruebas, fueron expresados en proporciones $y$ porcentajes, teniendo en consideración la cantidad de ocratoxina encontrada y comparando con el nivel máximo permitido en algunos países.

En el Cuadro 1 se muestra los resultados de las 62 muestras, observándose $35.5 \%$ de muestras contaminadas con ocratoxina, con promedio de $8 \mathrm{ug} / \mathrm{kg}$,
Cuadro 1. Resultados de las muestras de maíz amarillo, según estado de contaminación.

\begin{tabular}{lcc}
\hline \multicolumn{1}{c}{ Muestras } & N & $\%$ \\
\hline Contaminadas & 22 & 35.5 \\
No contaminadas & 40 & 64.5 \\
\hline Total & 62 & 100.00 \\
\hline
\end{tabular}

Estos resultados, son divergentes a lo informado en Brasil (Baldissera et al., 1994), EE.UU. (Price et al., 1993) y Europa (Basílico, 1995).

En el Cuadro 2 se muestra el nivel promedio de contaminación según el lugar de muestreo y el porcentaje de muestra que sobrepasa el nivel máximo permitido en algunos países.

Cuadro 2. Muestras de maíz amarillo según nivel de contaminación.

\begin{tabular}{lcc}
\hline $\begin{array}{c}\text { Lugar de } \\
\text { muestreo }\end{array}$ & $\begin{array}{c}\text { Promedio } \\
\text { ug/kg }\end{array}$ & $\begin{array}{c}\text { Mayor de } \\
5 \mathrm{ug} / \mathrm{kg}, \%\end{array}$ \\
\hline Granjas & 4.58 & 17.5 \\
Mercados & 12.54 & 40 \\
Barcos & 19.31 & 100 \\
\hline
\end{tabular}

Encontrándose mayor porcentaje en los barcos seguido de los mercados y las granjas, esta diferencia probablemente se deba a un mayor tiempo de almacenamiento durante su transporte y comercialización.

Existen diferencias entre los límites máximos permitidos en algunos países, que van de 5 a $50 \mathrm{ug} / \mathrm{kg}$ (FAO, 1997). Considerando el nivel de $5 \mathrm{ug} / \mathrm{kg}$ (ppb), el $27.4 \%$ de las muestras sobrepasa este nivel; sin embargo si se considera $50 \mathrm{ug} / \mathrm{kg}$, ninguna muestra sobrepasó este nivel. 
La gran diferencia entre los niveles máximos permitidos en los paises probablemente se deba a que países con alta incidencia de nefropatía porcina y posible enfermedad de la Balcanes adoptan medidas más rigurosas, como es el caso de los países del norte de Europa, por otro lado países donde no se presentan muchos casos de estas nefropatías o no se han realizado estudios adoptan los niveles más altos como es el caso de Brasil y Uruguay.

Por otro lado, si se toma en cuenta que $200 \mathrm{ug} / \mathrm{kg}$ es el nivel mínimo de ocratoxina A que produce efectos clínicos en el cerdo (Goodband et al., 1997) ninguna de las muestrạs evaluadas alcanzó estos niveles.

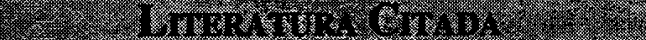

1. Baldissera, M. A.; J. M. Santurio, C. A. Mallmann; C. A. Almeida y $M$. Kipper. 1994. Aflatoxinas Zearalenona y Ocratoxina A en alimentos. I Congre- so Latinoamericano de Micotoxicología Brasil, p. 88-90.

2. Basílico, J.C. 1995. Micotoxinas en alimentos el riesgo sobre la mesa. Universidad Nacional del Litoral, Argentina, p. 53-57.

3. Bird, C. y R. Felman. 1996. Pruebas de inmunoensayo para la detección de micotoxinas. Alimentos Balanceados para Animales, USA, 3:30-32.

4. FAO. 1997. World wide regulations for mycotoxins. USA. 64:18-98.

5. Goodband, R.D.; M.D. Tokach; S.S. Dritz y J.L. Nelssen. 1997. General nutrition principles for swine. Kansas State University Agricultural Experiment Station, USA, p. 8.

6. Price, W.D.; R.A. Lovell y D.G. McChesney. 1993. Naturally occurring toxins in feedstuffs. J. Anim. Sci., USA, 71: 2556-2561. 